\title{
DISCUSSÃO DE ALTERNATIVAS NOS PROCESSOS DE AVALIAÇÃO AMBIENTAL ESTRATÉGICA EM MINAS GERAIS
}

\author{
DISCUSSION OF ALTERNATIVES IN STRATEGIC ENVIRONMENTAL ASSESSMENT \\ PROCESSES IN MINAS GERAIS - BRAZIL
}

\author{
Bruna Regina Melo CRESPO, Maria Rita Raimundo e ALMEIDA \\ Universidade Federal de Uberlândia - UFU. Emails: bruna2_melo@hotmail.com; mrralmeida@ufu.br

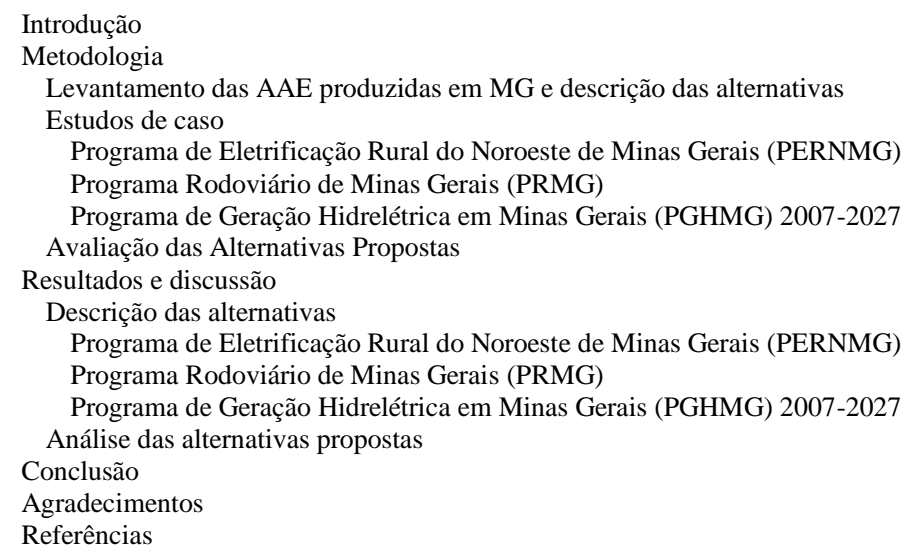

RESUMO - A Avaliação Ambiental Estratégica (AAE) é um instrumento de avaliação de impactos no âmbito de políticas, planos e programas, influenciando na formulação dos mesmos. No desenvolvimento da AAE, tem-se a discussão de alternativas estratégicas e sua análise detalhada é o principal mecanismo para garantir que a tomada de decisão seja ambientalmente adequada. No entanto, a falta de regulamentação do instrumento no Brasil tem feito com que as alternativas sejam desconsideradas. Diante disso, este trabalho propôs-se a avaliar o desenvolvimento de alternativas nas AAE praticadas no estado de Minas Gerais, sudeste do Brasil. Os relatórios destas AAE foram analisados de acordo com critérios de boas práticas. Dos três relatórios avaliados, apenas dois apresentaram estudo de alternativas e apenas um dos dois teve caráter estratégico. Uma vez que o estudo de alternativas não é realizado, ou o é de modo deficiente, o alcance da AAE como instrumento de gestão e planejamento ambiental fica reduzido.

Palavras-Chave - Avaliação Ambiental Estratégica, Alternativas, Níveis Estratégicos, Minas Gerais.

\begin{abstract}
Strategic Environmental Assessment (SEA) is an instrument of impact assessment applied in the context of policies, plans and programs (PPP), influencing their formulation. In the development of SEA, there is a discussion of strategic alternatives and its detailed analysis is the main mechanism to ensure that decision making sounds environmental. In Brazil, however, the alternatives are disregarded due lack of regulation of this instrument. In view of this, this paper proposes to evaluate the development of alternatives in SEA practiced in Minas Gerais State, in southeastern Brazil. The SEA reports were analyzed according to best practice criteria. Of the three reports evaluated, only two presented study of alternatives and just one presented strategic character. Since the study of alternatives is not performed, or in deficient mode, the SEA scope as an instrument for environmental planning and management is reduced.
\end{abstract}

Keywords - Strategic Environmental Assessment, Alternatives, Strategic Level, Minas Gerais State.

\section{INTRODUÇÃO}

Ao realizar um retrospecto das políticas públicas adotadas em diversos países, nota-se que muitas tiveram como efeitos colaterais impactos socioambientais negativos (Sánchez, 2008). Somado a isso, usualmente, a Avaliação de Impactos Ambientais (AIA) aplicada a projetos não consegue considerar satisfatoriamente impactos indiretos ou impactos cumulativos. Além disto, no momento em que a AIA de projetos é realizada muitas decisões já foram tomadas, reduzindo as possibilidades de se considerar outras alternativas tecnológicas e locacionais. Surge, então, a necessidade de um instrumento que antecipe as questões ambientais dentro do processo decisório, como a Avaliação
Ambiental Estratégica (AAE). Sánchez (2008) exemplifica que se uma empresa solicita uma licença para construção de uma usina termoelétrica a gás, o Estudo de Impacto Ambiental (EIA) não considerará e, portanto, não avaliará o impacto causado por outras alternativas: isto acontece porque quando o EIA é solicitado o projeto já foi delineado e sua viabilidade econômica atestada, de modo que a AAE se torna o melhor cenário para a análise de alternativas. O Ministério do Meio Ambiente (MMA) destaca a importância do caráter contínuo da avaliação da qualidade do meio ambiente e assinala a necessidade de integrar os aspectos biofísicos, aspectos geofísicos, 
econômicos, sociais e políticos à tomada de decisão, o quanto antes (MMA, 2002), além de questões como, por exemplo, contaminação de solos e subsolos, de aquíferos e estuários e ar atmosférico, entre outros.

Nesse contexto, AAE é o nome dado a todo processo de avaliação de impactos que se realiza em âmbito mais amplo que aquela realizada para projetos individuais (Sánchez, 2008). Para Partidário (2007), a AAE é um instrumento de avaliação de impactos de caráter estratégico que busca promover o desenvolvimento sustentável por meio da avaliação de riscos e oportunidades pertinentes à proposição e execução das Políticas, Planos e Programas (PPP). O caráter estratégico pode ser entendido como um conjunto coerente de grandes prioridades e de decisões que orientam o desenvolvimento e a construção do futuro de uma organização (neste caso, do próprio governo) em um horizonte de longo prazo (Porto, 1998).

Ainda, o papel da AAE vai além da verificação dos potenciais impactos, ela deve influenciar a formulação desses PPP. Sánchez (2008) ressalta que o principal ganho da AAE está na capacidade de influenciar a formulação dos PPP, pois não é um instrumento que visa aprovar ou não os PPP ou verificar suas consequências após a sua formulação, sendo, portanto, um instrumento de caráter proativo. Assim, caberia a AAE integrar os critérios ambientais à formulação de PPP, possibilitando a identificação de riscos e a proposição de alternativas (Thérivel \& Partidário, 1996).

No entanto, cabe destacar que a AAE não deve ser vista como um substituto ou como um instrumento mais eficaz que a AIA de projetos; o uso da AAE em estágios anteriores à AIA de projetos pode promover uma avaliação de projetos mais rápida e eficiente, além de levar a integração de PPP e facilitar abordagem de impactos cumulativos e indiretos (Sánchez, 2008).

$\mathrm{Na}$ literatura existe um consenso de que a AAE se torna útil por dois motivos principais: sua capacidade de superar as limitações do processo de AIA e por ser um instrumento de promoção da sustentabilidade por meio da integração de aspectos ambientais, sociais e econômicos (Sánchez, 2008; Egler, 2011). Logo, o papel da AAE é o de promover um desenvolvimento sustentável por meio da inclusão da questão ambiental no processo decisório (Oliveira et al., 2009). Na legislação que obriga e regulamenta a prática da $\mathrm{AAE}$ na Europa, Diretiva Europeia 2001/42/CE, a AAE constitui um instrumento importante de integração das considerações ambientais na preparação e aprovação de determinados planos e programas que possam ter efeitos significativos no ambiente, uma vez que garante que os efeitos ambientais da aplicação dos planos e programas sejam levados em consideração durante a sua preparação e antes da sua aprovação (JOCE, 2001).

Como fechamento de tudo isso, de acordo com Fischer (2007), a AAE tem três aspectos básicos, por definição: é um processo sistemático de suporte à decisão, com o intuito de garantir que o meio ambiente e outros aspectos de sustentabilidade sejam considerados na elaboração de PPP; é um instrumento baseado em evidências, com o intuito de fornecer rigor científico à elaboração de PPP por meio de técnicas e métodos de avaliação; e trata-se de um instrumento de apoio à decisão e incentivo ao desenvolvimento sustentável e à governança, por meio do estabelecimento de um foco, por exemplo, nas questões e alternativas a serem consideradas de forma sistemática em diferentes âmbitos e níveis de planejamento.

Com o intuito de introduzir a sustentabilidade nas proposições dos PPP, a AAE conta com uma série de etapas. $O$ desenvolvimento de alternativas estratégicas está entre estas etapas, conforme apontam Oliveira et al. (2009) e Egler (2011). No caso dos primeiros autores, ainda é destacado que na AAE busca-se desenvolver alternativas estratégicas que levem a uma opção ambientalmente menos nociva, mas que possa atender aos objetivos propostos (Oliveira et al., 2009). Devem ser identificadas alternativas de investimento, localização de ações e projetos, emprego de tecnologia para a PPP em análise, pensando sempre em qual é a melhor opção, ou seja, aquela que permita o melhor aproveitamento dos recursos ambientais gerando menor impacto e, ao fim, alcance o mesmo objetivo (MMA, 2002).

Alguns outros autores também destacam a importância das alternativas no contexto de desenvolvimento da AAE. Silva (2010) ressalta que o processo de AAE deve facilitar a identificação das possibilidades de desenvol- 
vimento e das alternativas que sejam mais sustentáveis. Sánchez (2008) e Egler (2011) nos mostram que, no processo de $\mathrm{AAE}$, as alternativas são mais variadas, pois no estágio de planejamento há poucas limitantes ou nenhuma; no estágio de projetos, por sua vez, muitas decisões já foram tomadas restringindo o número de alternativas. Egler (2011) afirma também que o tempo age de modo flexível na análise de PPP, possibilitando um maior refinamento das alternativas. De acordo com Ganem et al. (2014), na AAE existe possibilidade de apreciar um número maior de alternativas, visto que ela se insere numa fase em que ainda não há muitas decisões tomadas. Com um maior leque de alternativas torna-se mais fácil reduzir ou evitar impactos negativos.

Assim, é notório que a análise detalhada de prováveis alternativas é o principal mecanismo de garantir que a tomada de decisão seja a mais adequada do ponto de vista ambiental. No entanto, é a previsão dos impactos causados que permite que as alternativas sejam comparadas e que, assim, o processo de tomada de decisão ocorra com base na comparação dos aspectos positivos e negativos de cada alternativa, sejam fatores ambientais, políticos e socioeconômicos (MMA, 2002).

No contexto brasileiro, a prática da AAE é recente quando comparada aos países desenvolvidos e incentivada pelo Banco Mundial e pelo Banco Interamericano de Desenvolvimento, principalmente, por meio da inserção de requisitos ambientais ao processo de financiamento de ações de desenvolvimento, além de ações de capacitação (Pellin et al., 2011). O que emerge desse cenário é a não existência de legislação que regulamente a AAE e isto tem contribuído para uma prática questionável e dissociada de princípios ou diretrizes básicos (Pellin et al., 2011).

Ainda, alguns estados brasileiros adotaram em suas legislações algumas referências a utilização da AAE. Este é o caso do estado de Minas Gerais que editou dois decretos: o Decreto Estadual no 43.372/2003, que cria o Núcleo de Gestão Ambiental (NGA); e o Decreto Estadual $\mathrm{n}^{\mathrm{o}}$ 45.761/2011, que altera dispositivos do decreto anterior.

De acordo com esses dispositivos, os NGA foram criados com o intuito de incorporar as políticas ambientais no desenvolvimento de políticas públicas setoriais e suas funções seriam, entre outras: assessorar os secretários de estado, com o intuito de inteirá-los acerca das decisões do Conselho de Política Ambiental (COPAM) que interfiram, de alguma maneira, nas PPP propostas pelas secretarias; definir o Termo de Referência (TR) de cada PPP; e coordenar, em parceria com a Secretaria de Estado de Meio Ambiente e Desenvolvimento Sustentável (SEMAD), a elaboração das AAE (Minas Gerais, 2003). Nesse contexto, algumas AAE foram produzzidas em Minas Gerais.

É interessante notar que, apesar de referências ao uso da $\mathrm{AAE}$, nenhuma diretriz foi dada quanto à forma como ela deve ser praticada e, muito menos, como deve ser realizado o estudo de alternativas. Assim, o presente estudo propõe avaliar como as alternativas são identificadas e propostas dentro da AAE praticada no Brasil, mais especificamente naquela praticada no estado de Minas Gerais.

\section{METODOLOGIA}

\section{Levantamento das AAE produzidas em MG e descrição das Alternativas}

Para identificar as AAE realizadas em Minas Gerais foi feita uma busca na página eletrônica da Secretária de Estado de Meio Ambiente e Desenvolvimento Sustentável do estado de Minas Gerais (SEMAD) e uma pesquisa na internet em geral, utilizando o site de busca Google (www.google.com.br). No site de busca foram realizadas duas varreduras, uma no mês de agosto/2016 e outra no mês de novembro/2017, sendo esta última para verificação de que se não haveria mais nenhuma AAE sido realizada após a primeira busca e para finalização do presente trabalho. $\mathrm{Na}$ primeira procura, utilizando como palavras-chave: "avaliação ambiental estratégica em Minas Gerais", houve aproximadamente 506.000 resultados em agosto/2016 e 504.000 resultados em novembro/2017. A segunda busca foi realizada com as palavras-chave: "relatório de avaliação ambiental estratégica em Minas Gerais", produzindo aproximadamente 299.000 resultados em agosto de 2016 e 316.000 resultados em novembro de 2017. Na página 
eletrônica da SEMAD (www.semad.mg. gov.br) foi realizada uma busca em agosto/2016, utilizando a palavra-chave: "aae", sendo encontrados 21 resultados e uma busca em novembro/2017, com a mesma palavra chave, sendo encontrados 26 resultados.

A partir de todos os resultados encontrados nas diferentes buscas, foi possível identificar três AAE produzidas no estado de Minas Gerais que, consequentemente, foram selecionadas para estudo neste trabalho, são elas: 1) Avaliação Ambiental Estratégica do Programa de Eletrificação Rural do Noroeste de Minas Gerais, 2) Avaliação Ambiental Estratégica do Programa Rodoviário de Minas Gerais e 3) Avaliação Ambiental Estratégica do Programa de Geração Hidrelétrica em Minas Gerais 20072027. Apesar das páginas eletrônicas citarem estas AAE, em nenhuma delas foi possível ter acesso ao relatório de AAE na íntegra. No entanto, notou-se que essas mesmas AAE identificadas na busca estavam entre aquelas levantadas no trabalho de Malvestio (2013), sendo os relatórios aqui utilizados de AAE disponibilizados por esta autora.

Para a descrição das alternativas, em cada uma das três AAE selecionadas fez-se a narrativa de como a questão das alternativas foi trabalhada com base nas informações apresentadas nos relatórios de AAE.

\section{Estudos de Caso}

A seguir, encontra-se uma breve descrição, a partir das informações contidas nos respectivos relatórios, do que são os programas, em que contexto a AAE foi realizada e uma síntese do conteúdo do relatório de $\mathrm{AAE}$ dos três que são os estudos de caso desse trabalho.

\section{Programa de Eletrificação Rural do Noroeste de Minas Gerais (PERNMG)}

O Programa de Eletrificação Rural do Noroeste de Minas Gerais (PERNMG) tem por objetivo implantar rede de transmissão e distribuição de energia elétrica, integradas a subestações e a usina hidrelétrica de Queimado, visando levar energia à zona rural e cumprir metas de programas sociais do estado de Minas Gerais como "Luz para todos" e "Clarear".

$\mathrm{O}$ documento que contém o relatório da AAE foi elaborado para fornecer informações ao Banco Interamericano de Desenvolvimento com a intenção de conseguir, junto ao Banco, financiamento para as obras do programa. De tal forma, ele apresenta de forma detalhada as características socioeconômicas, do meio físico e biológico dos municípios que integram a região, bem como as obras e atividades a serem realizadas ao longo do programa, seus potenciais impactos e respectivas ações mitigadoras. Outro ponto tratado no relatório de AAE é a capacidade de gestão ambiental da Companhia Energética de Minas Gerais (Cemig) e da Secretaria de Estado de Desenvolvimento Econômico (SEDE), corresponsáveis pela implantação do programa. Por fim, é apresentado o plano de controle dos impactos ambientais causados.

Programa Rodoviário de Minas Gerais (PRMG)

A Secretaria de Estado de Transportes e Obras Públicas (SETOP) e o Departamento de Estradas de Rodagem (DER/MG), visando à adequação da malha rodoviária mineira para atender a demanda de transporte, até 2016, estruturaram o Programa Rodoviário de Minas Gerais (PRMG).

Dentre os objetivos do PRMG estão: a melhoria da acessibilidade a mercados, aos serviços sociais, a mobilidade dos habitantes mineiros, além da integração das diversas regiões de planejamento do Estado, reduzindo distâncias e custos de transporte. O PRMG abrange obras rodoviárias de características técnicas e tipologias diversas, distribuídas em 195 trechos que totalizam cerca de $14 \mathrm{mil}$ quilômetros de extensão. Estes trechos são divididos segundo a extensão e jurisdição em quatro categorias: federais $(8.051,5 \mathrm{~km}$ $57,21 \%$ ), estaduais $(3.314,9 \mathrm{~km}-23,56 \%)$, municipais $(2.059,20 \mathrm{~km}-14,63 \%)$ e mistas $(647,0 \mathrm{~km}-4,60 \%)$.

$\mathrm{O}$ relatório de AAE apresenta em seu conteúdo uma análise das interações do PRMG com o Plano Mineiro de Desenvolvimento Integrado (PMDI) e o Plano Plurianual de Ação Governamental (PPAG) - 2004/2007, bem como a legislação relativa ao licenciamento ambiental de rodovias, as interações com Unidades de Conservação e intervenções em Áreas de Preservação Permanente. Ainda, foi feita a análise dos componentes da área de estudo e projeções de um cenário futuro com e sem a intervenção pretendida. De acordo com o relatório, a AAE tornou possível a proposição de alternativas locacionais que sejam ambientalmente mais sustentáveis e continuem atendendo os objetivos do programa. 
Programa de Geração Hidrelétrica em Minas Gerais (PGHMG) 2007-2027

O governo do Estado de Minas Gerais, por meio do Programa de Geração Hidrelétrica em Minas Gerais (PGHMG) 2007-2027, tem por objetivo prover a demanda de energia do Estado a partir de seu próprio parque gerador, além de expandi-lo para também gerar excedentes exportáveis. A Secretaria de Estado de Desenvolvimento Econômico (SEDE) estabeleceu que o rol de empreendimentos para alcançar este objetivo deverá ser composto por 45 Usinas Hidrelétricas (UHE) e 355 Pequenas Centrais Hidrelétricas $(\mathrm{PCH})$. UHE são hidrelétricas com mais de $30 \mathrm{MW}$ de potência instalada e reservatórios maiores que $3 \mathrm{~km}^{2}$, enquanto as PCH têm potência instalada potência superior a 1 MW e igual ou inferior a 30 MW e reservatórios de no máximo $3 \mathrm{~km}^{2}$. O relatório apresenta o plano de trabalho para a realização da AAE, assim como o planejamento elétrico nacional e mineiro, a gestão dos recursos hídricos e, posteriormente, um estudo do meio físico, dos ecossistemas e do meio socioeconômico a ser afetado pelo pelos empreendimentos do programa. Outros dados importantes são as projeções da demanda futura da população por energia, para que se possa saber quanto à capacidade de geração de energia precisa aumentar.

\section{Avaliação das Alternativas Propostas}

Depois de realizada a coleta de informações nos relatórios de AAE, buscou-se parâmetros para a interpretação dos dados obtidos, a fim de verificar como foram elaborados os estudos de alternativas.

Inicialmente, buscou-se avaliar o caráter estratégico das alternativas propostas. Para esse fim, recorreu-se ao que a International Association for Impact Assessment (IAIA) propõe. De acordo com a IAIA (2014), as diferentes formas de alcançar os objetivos de políticas, planos e programas ocupam papel central na AAE; as alternativas devem fazer parte da construção dos PPP e serem desenvolvidas de forma sistemática e clara, podendo enquadrar-se em pelo menos um dos sete tópicos: estratégico, orientados para valores, orientados para efeitos, setorial, espacial, modal ou temporal. O que vem a ser cada um desses tópicos é trazido em detalhes por EPA (2015), conforme o apresentado na tabela 1 .

Tabela 1 - Critérios de avaliação das alternativas de AAE. Fonte: adaptado de EPA (2015).

\begin{tabular}{|c|c|c|}
\hline $\begin{array}{c}\text { Tipo de } \\
\text { alternativa }\end{array}$ & Descrição & Exemplo \\
\hline Estratégico & $\begin{array}{l}\text { Opções de alto nível que atingem um } \\
\text { determinado objetivo. Este tipo normalmente é } \\
\text { realista apenas em nível de política. }\end{array}$ & $\begin{array}{l}\text { No caso da gestão de resíduos, as opções estratégicas } \\
\text { considerariam o aterro sanitário versus a incineração. }\end{array}$ \\
\hline $\begin{array}{l}\text { Orientado } \\
\text { para valores }\end{array}$ & $\begin{array}{l}\text { Alternativas que abordam prioridades políticas, } \\
\text { valores culturais ou questões de segurança. }\end{array}$ & $\begin{array}{c}\text { Para a gestão de bacias hidrográficas, as alternativas podem } \\
\text { concentrar-se na redução do risco público ou na melhoria da } \\
\text { qualidade das águas. }\end{array}$ \\
\hline $\begin{array}{c}\text { Orientado } \\
\text { para efeitos }\end{array}$ & $\begin{array}{l}\text { Alternativas que tratam de questões } \\
\text { identificadas durante o scooping. Essas } \\
\text { alternativas são eficazes para mitigar potenciais } \\
\text { efeitos significativos e particularmente úteis } \\
\text { para evitar impactos na natureza. }\end{array}$ & $\begin{array}{l}\text { Para planos locais de uso da terra onde a inundação é um } \\
\text { problema, alternativas poderiam incluir diferentes formas de } \\
\text { evitar novas inundações ou lidar com o evento. }\end{array}$ \\
\hline Setoriais & $\begin{array}{l}\text { Alternativas que analisam a viabilidade e as } \\
\text { necessidades setoriais. No nível estratégico, as } \\
\text { políticas podem ser formuladas para promover } \\
\text { um setor versus outro. }\end{array}$ & $\begin{array}{l}\text { No sector das energias renováveis, as alternativas são } \\
\text { frequentemente baseadas em considerações de tecnologia- } \\
\text { custo-infraestrutura. }\end{array}$ \\
\hline Espaciais & $\begin{array}{l}\text { Alternativas locacionais para a implementação } \\
\text { de objetivos de planejamento. Este tipo de } \\
\text { alternativa é particularmente relevante em nível } \\
\text { local. }\end{array}$ & $\begin{array}{l}\text { As alternativas espaciais são comumente usadas no } \\
\text { planejamento do uso da terra, onde são exploradas várias } \\
\text { localizações para desenvolvimento residencial, industrial e } \\
\text { comercial. Similarmente, a localização rural versus urbana } \\
\text { de alternativas é plausível para PPP. }\end{array}$ \\
\hline Modais & $\begin{array}{l}\text { Diferentes alternativas tecnológicas pata atingir } \\
\text { o mesmo objetivo. }\end{array}$ & $\begin{array}{l}\text { Para a gestão de resíduos, as alternativas poderiam incluir a } \\
\text { promoção da reciclagem e utilização eficiente dos materiais, } \\
\text { bem como opções entre incineração e aterro sanitário. }\end{array}$ \\
\hline Temporais & $\begin{array}{l}\text { Alternativas para o calendário de } \\
\text { implementação de medidas do PPP. }\end{array}$ & $\begin{array}{c}\text { As alternativas temporais são úteis para garantir que a } \\
\text { infraestrutura seja fornecida antes do desenvolvimento e } \\
\text { que a sequência deste corresponda às capacidades e } \\
\text { necessidades do serviço local. }\end{array}$ \\
\hline
\end{tabular}


Assim, as informações apresentadas na tabela 1 foram utilizadas como referência para a avaliação de cada tópico. As alternativas propostas nos relatórios de AAE foram avaliadas em enquadrase ou não se enquadra para cada um dos tópicos apresentados. Lembrando que o desenvolvimento de alternativas de AAE para ser considerado estratégico deve ser realizado de modo que as alternativas possam se enquadrar em, ao menos, um dos sete tópicos apresentados (Carvalho, 2014; IAIA, 2014).

Complementando a análise do estudo de alternativas realizada neste trabalho, também foram avaliados os critérios de boas práticas organizados por Carvalho (2014) para verificar o desenvolvimento da proposição de alternativas naquelas AAE que tiveram este estudo. Esta referência foi utilizada pelo fato do autor já ter realizado uma ampla revisão de literatura e recorrido a vários outros autores para fazer a sua proposição.

De acordo com Carvalho (2014), para garantir o desenvolvimento de alternativas estratégicas o processo de AAE deve:

- Auxiliar na identificação das melhores alternativas (IAIA, 2002, segundo Carvalho,
2014) existentes possíveis, mais sustentáveis e de longo prazo (Therrivel, 2010, segundo Carvalho, 2014).

- Oferecer oportunidades de proposição de alternativas tanto pelas autoridades ambientais ou de sustentabilidade, como por outras instituições especializadas ou pelo público (Therrivel, 2010, segundo Carvalho, 2014).

- Descrever como as alternativas foram desenvolvidas (Therrivel, 2010, segundo Carvalho, 2014).

- Apresentar uma avaliação comparativa das alternativas "mais razoáveis" (Jones et al., 2005, segundo Carvalho, 2014).

- Documentar como as alternativas preferidas foram escolhidas (Therrivel, 2010, segundo Carvalho, 2014).

- Apresentar um resumo das razões para a seleção das alternativas consideradas, incluindo qualquer dificuldade na compilação da informação requerida (JOCE, 2001 segundo Carvalho, 2014).

Esses critérios foram aplicados ao estudo de alternativas de cada relatório de AAE e avaliados em cumpriu e não cumpriu.

\section{RESULTADOS E DISCUSSÃO}

A identificação e o desenvolvimento de alternativas são etapas importantes do processo de AAE, pois permitem que a melhor decisão seja tomada (MMA, 2002). Para que se possa compreender como as alternativas dos relatórios de AAE foram avaliadas nos estudos de caso, inicialmente, será apresentado sucintamente como o estudo de alternativas foi realizado em cada AAE de acordo com as informações apresentadas nos relatórios de AAE e, em seguida, serão apresentados os resultados da análise realizada.

\section{Descrição das Alternativas}

A seguir, encontra-se uma breve descrição das alternativas propostas em cada uma das três AAE analisadas, de acordo com o relatório de AAE.

Programa de Eletrificação Rural do Noroeste de Minas Gerais (PERNMG)

As informações contidas no relatório de AAE não apresentam discussão de alternativas, sejam elas locacionais, tecnológicas ou de qualquer outro tipo. O único cenário estudado é o da execução de todas as obras do programa. O conjunto de obras do programa inclui a construção de três subestações e a ampliação de outras seis subestações, construção de 162,13 quilômetros de linhas de transmissão e a implantação de 1367 quilômetros de redes de distribuição rural.

\section{Programa Rodoviário de Minas Gerais (PRMG)}

Nessa AAE, foram escolhidos dois cenários futuros possíveis. Um tendencial, que foi projetado com base na dinâmica econômica e o no crescimento vivenciado no momento da construção da AAE (julho de 2007).

Um segundo cenário, chamado de "Conquista do melhor futuro", foi eleito pela Secretaria de Estado de Transportes e Obras Públicas (SETOP) dentre as quatro situações futuras possíveis de acordo com Plano Mineiro de Desenvolvimento Integrado (PMDI). Esse cenário prevê um contexto econômico mundial e nacional favorável, assim Minas Gerais aproveitaria as oportunidades para dar início a um longo período de crescimento econômico, aumento dos índices educacionais, redução da pobreza e da desigualdade e uso sustentável dos recursos ambientais. 
O PRMG abrange um conjunto de obras que juntas somam cerca de 14 mil quilômetros de extensão, divididos em 95 trechos. Cuja implantação é analisada de duas maneiras, sendo que estas alternativas são examinadas nos dois possíveis cenários futuros descritos anteriormente:

i) com a implementação de $100 \%$ das obras previstas;

ii) uma variante que consiste na execução das intervenções previstas no Plano Estadual de Logística de Transporte do Estado de Minas Gerais (PELT), no qual haveria a redução de $13 \%$ das "Rodovias Turístico-Ecológicas" e $29 \%$ do Subprograma "Complemento da Rede Rodoviária", em relação ao conjunto de obras propostas pelo PRMG.

A carteira de projetos do PELT foi escolhida como possível alternativa, pois o plano objetiva o desenvolvimento da multimodalidade, gerando ganhos socioambientais e econômicos, reduzindo impactos ambientais, fortalecendo as empresas, tornando os produtos regionais mais competitivos e assim aumentando a qualidade de vida da população.

Programa de Geração Hidrelétrica em Minas Gerais (PGHMG) 2007-2027

O Programa de Geração Hidrelétrica em Minas Gerais prevê a ampliação do seu parque gerador para o atendimento da demanda energética futura e possível exportação de energia para outras regiões do país. A Secretaria de Estado de Desenvolvimento Econômico (SEDE) prevê a implantação de 45 Usinas Hidrelétricas (UHE) e 335 Pequenas
Centrais Hidrelétricas (PCH). Para esta ampliação sugeriu-se quatro cenários:

- um cenário de expansão do parque gerador com todos os empreendimentos - C I;

- três cenários de expansão do parque gerador com crescentes graus de complexidade socioambiental - C II, CIII e CIV.

O primeiro cenário $(\mathrm{C}$ I) de expansão do parque gerador permite que todos os empreendimentos sejam implantados, ele tem baixo nível de restrição do ponto de vista socioambiental e funciona com um exercício para que se possam entender os limites da capacidade de geração do estado.

Os demais cenários (C II, CIII e CIV) foram formulados levando em consideração valores limites para o Índice de Impacto Ambiental (IA). Cada um dos empreendimentos previstos pelo programa foi caracterizado mediante seus índices de impacto ambiental, benefício socioeconômico e energético.

Os valores de IA de cada empreendimento foram organizados em um gráfico de maneira crescente, originando uma curva, como apresentado na figura 1 . O relatório de AAE não descreve em detalhes como o IA foi calculado, no entanto, esta omissão não atrapalha o entendimento geral de como as opções de alternativas foram criadas. Ainda, foram estabelecidas cinco classes para o Índice de Impacto Ambiental (Tabela 2), realizando os cortes por meio do critério de desvio em relação à média (por meio de uso de software de georreferenciamento).

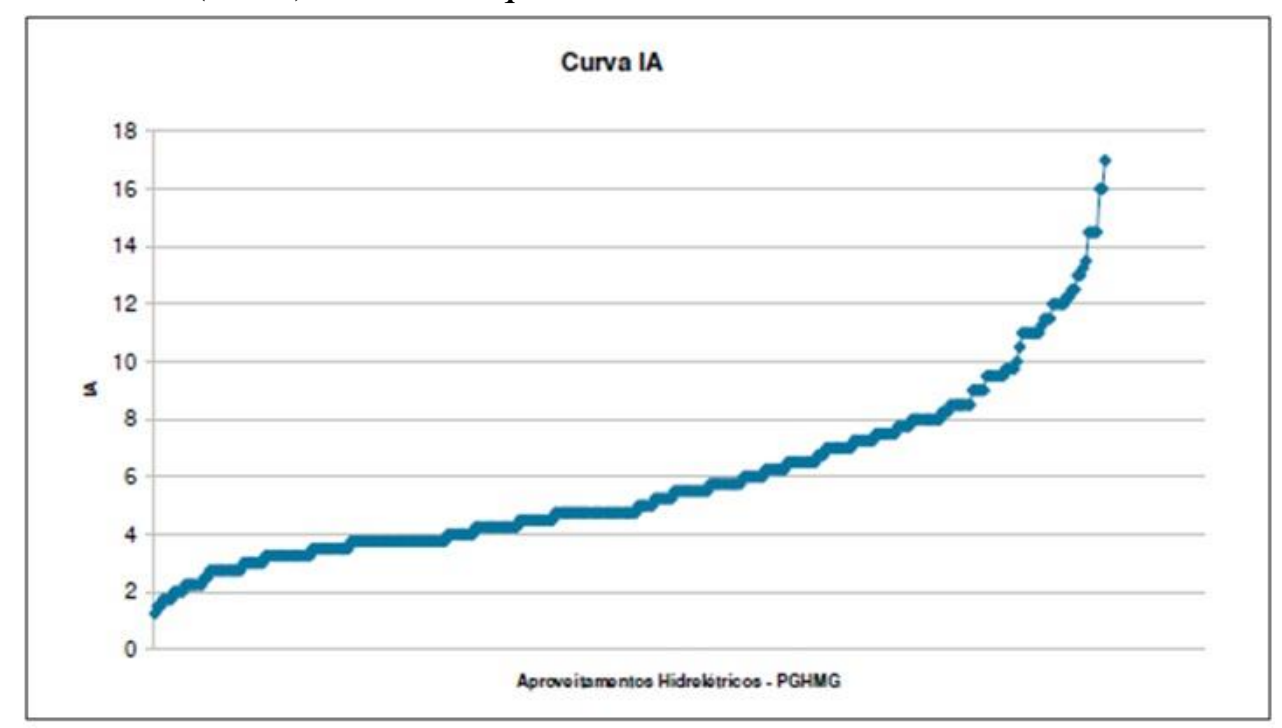

Figura 1 - Variação dos valores de Índice de Impacto Ambiental (IA) em função dos aproveitamentos hidrelétricos. Fonte: Relatório da Avaliação Ambiental Estratégica do Programa de Geração Hidrelétrica de Minas Gerais. 
Tabela 2 - Classes do Índice de Impacto Ambiental (IA). Fonte: relatório da Avaliação Ambiental Estratégica do Programa de Geração Hidrelétrica de Minas Gerais.

\begin{tabular}{c|c|c}
\hline \multicolumn{2}{c|}{ Classes do IA Geral } & $\mathbf{N}^{\mathbf{0}}$ de Empreendimentos \\
\hline MA Muito Alto & $-13,08$ a $-17,00$ & 9 (UHEs) \\
\hline A Alto & $-10,15$ a $-13,07$ & $22(5$ PCHs + 17 UHEs $)$ \\
\hline M Médio & $-7,26$ a $-10,16$ & $52(39$ PCHs +13 UHEs $)$ \\
\hline B Baixo & $-4,35$ a $-7,25$ & $131(128$ PCHs + 3 UHEs $)$ \\
\hline MB Muito Baixo & 0 a $-4,34$ & $166(163$ PCHs + 3UHEs $)$ \\
\hline
\end{tabular}

Assim os cenários possíveis foram definidos com base na curva do índice de Impacto Ambiental e nas classes de IA, sendo eles:

- um segundo cenário que contém todos os empreendimentos do PGHMG até o valor do IA de 13,25 (CII);

- um terceiro cenário que contém todos os empreendimentos até o valor do IA de 10, 5 (CIII);

- um quarto cenário que contém todos os empreendimentos até o valor do IA de 7,25 (CIV).

Em seguida, procedeu-se a identificação da demanda futura de energia. Para tanto, utilizouse os cenários do Plano Mineiro de Desenvolvimento Integrado (PMDI) I e III, respectivamente, "Conquista do melhor futuro" e "Superação de adversidades".

No cenário "Conquista do melhor futuro", os contextos internacional e nacional são favoráveis, permitindo que o estado de Minas Gerais aproveite as oportunidades iniciando um período duradouro de crescimento econômico, redução das desigualdades sociais e uso sustentável dos ativos ambientais. Por sua vez, no cenário "Superação de adversidades", os contextos internacional e nacional são desfavoráveis, com baixas taxas de crescimento econômico e manutenção das desigualdades. Nesse último cenário, Minas Gerais aproveita as raras oportunidades, utilizando a inovação para alcançar um crescimento acima da média nacional.

As informações dessas projeções são correlacionadas com cada cenário do parque gerador do estado de Minas Gerais buscando encontrar o cenário de maior potencial de geração e menor impacto socioambiental.

\section{Análise das Alternativas Propostas}

Como visto anteriormente, o Programa de Eletrificação Rural do Noroeste de Minas Gerais não apresentou um estudo para a discussão de alternativas para sua implantação, impossi- bilitando sua análise.

O Programa Rodoviário de Minas Gerais apresentou como alternativa a execução das obras previstas pelo Programa Estadual de Logística e Transporte do Estado de Minas Gerais, no qual haveria uma redução de $13 \%$ das "Rodovias Turístico-Ecológicas" e 29\% do Subprograma "Complemento da Rede Rodoviária”, em relação à proposta original do PRMG. Analisando este estudo de alternativas a luz dos tópicos apresentados, têm-se os resultados apresentados na tabela 3. Desses resultados, verificou-se que a AAE do PRMG não se enquadrou em nenhuma categoria que pudesse lhe dar um caráter estratégico (estratégico, orientados para valores, orientados para efeitos, setorial, espacial, modal ou temporal), não tendo, portanto, esse caráter. $\mathrm{O}$ Programa de Geração Hidrelétrica de Minas Gerais 2007-2027 apresenta quatro cenários alternativos, com base no índice de impacto ambiental, sendo:

- um cenário de expansão do parque gerador com todos os empreendimentos (CI);

- um segundo cenário que contém todos os empreendimentos do PGHMG até o valor do IA de 13,25 (CII);

- um terceiro cenário que contém todos os empreendimentos até o valor do IA de 10, 5 (CIII);

- um quarto cenário que contém todos os empreendimentos até o valor do IA de 7,25 (CIV).

Analisando as alternativas apresentadas com base nos indicadores têm-se os resultados apresentados na tabela 4. Desse modo, pode-se dizer que, de acordo com os critérios apresentados pela IAIA, as alternativas apresentadas no Programa de Geração Hidrelétrica de Minas Gerais alcançaram seu papel estratégico, pois se enquadram no tópico orientado para efeitos. Cabe ressaltar que, embora $\mathrm{o}$ relatório de $\mathrm{AAE}$ indique a 
necessidade de estudos sobre a geração de energia a partir de outras fontes, elas não são tratadas como alternativas pela AAE, não se enquadrando, portanto, no tópico "Modais".

Tabela 3 - Resultados da análise do caráter estratégico das alternativas propostas na AAE do Programa Rodoviário de Minas Gerais,

\begin{tabular}{c|l}
\hline $\begin{array}{c}\text { Tipos de } \\
\text { alternativa }\end{array}$ & \multicolumn{1}{c}{ Alternativa em análise } \\
\hline Estratégico & $\begin{array}{l}\text { Não se enquadra. Encontra-se em um nível em que a maioria das decisões de planejamento já foi } \\
\text { tomada. Além de que não foi avaliada a opção de outros modais de transporte. }\end{array}$ \\
\hline $\begin{array}{c}\text { Orientado para } \\
\text { valores }\end{array}$ & $\begin{array}{l}\text { Não se enquadra. Não incluem valores culturais, ambientais, redução de riscos, prioridades } \\
\text { políticas. }\end{array}$ \\
\hline $\begin{array}{c}\text { Orientado para } \\
\text { efeitos }\end{array}$ & Não se enquadra. Não prevê a mitigação de efeitos significativos identificados durante o estudo. \\
\hline Setoriais & $\begin{array}{l}\text { Não se enquadra. O programa aborda apenas o setor de transportes e as alternativas se referem } \\
\text { apenas ao transporte rodoviário. }\end{array}$ \\
\hline Espaciais & Não se enquadra. Não inclui opções para a localização das rodovias. \\
\hline Modais & Não se enquadra. Não cogita a implementação de outro tipo de transporte, apenas o rodoviário. \\
\hline Temporais & Não se enquadra. Não apresenta outras propostas para execução das obras. \\
\hline
\end{tabular}

Tabela 4 - Resultados da análise do caráter estratégico das alternativas propostas na AAE do Programa de Geração Hidrelétrica de Minas Gerais.

\begin{tabular}{c|l}
$\begin{array}{c}\text { Tipos de } \\
\text { alternativa }\end{array}$ & \multicolumn{1}{c}{ Alternativa em análise } \\
\hline $\begin{array}{c}\text { Estratégico } \\
\text { Orientado para } \\
\text { valores }\end{array}$ & $\begin{array}{l}\text { Não se enquadra. Tem caráter menos abrangente. Muitas decisões já foram tomadas. Além de } \\
\text { que não foram avaliadas outras opções de geração de energia. }\end{array}$ \\
\hline $\begin{array}{c}\text { Orientado para } \\
\text { efeitos }\end{array}$ & $\begin{array}{l}\text { Enquadra-se. Visa reduzir efeitos significativos causados pelo programa, principalmente } \\
\text { impactos ambientais. }\end{array}$ \\
\hline $\begin{array}{c}\text { Setoriais } \\
\text { Espaciais }\end{array}$ & $\begin{array}{l}\text { Não se enquadra. O programa aborda apenas a questão energética e as alternativas tratam apenas } \\
\text { da energia hidrelétrica. }\end{array}$ \\
\hline cada empreendimento apresentado pelo programa. \\
\hline Memporais & Não se enquadra. Aborda apenas a geração de energia a partir de hidrelétricas. \\
\hline
\end{tabular}

Em relação a como as alternativas foram elaboradas, utilizando os critérios reunidos por Carvalho (2014), o desenvolvimento de alternativas foi avaliado nos relatórios de Programa Rodoviário de Minas Gerais (PRMG) e no Programa de Geração Hidrelétrica de Minas Gerais (Tabela 5), já que o Programa de Eletrificação Rural do Noroeste de Minas Gerais (PGHMG) não apresentou qualquer tipo de estudo de alternativas.

De acordo com a avaliação da tabela 5, o relatório da AAE do PRMG não cumpriu nenhum dos critérios de boas práticas para o desenvolvimento de alternativas. A segunda alternativa proposta, ou seja, a realização do conjunto de obras previsto no PELT, foi apenas apresentada como uma redução em relação ao total de obras previstas pela primeira alternativa do PRMG.

Deste modo, pode-se dizer que, mesmo apresentando duas opções, o estudo de alternativas não seguiu nenhuma orientação de boas práticas, não sendo, portanto, um desenvolvimento de alternativas adequado.

Já a AAE do PGHMG apresenta de forma detalhada a previsão de crescimento de demanda do setor energético do estado de Minas Gerais até 2027 e avalia as possibilidades de alteração dessa demanda em diferentes cenários de desenvolvimento propostos para o estado. Os cenários são submetidos a uma análise comparativa em que a capacidade de atender a demanda energética futura e o impacto socioeconômico causado também são incluídos como critérios. Durante a construção da AAE, houve reuniões públicas que são oportunidades para que organizações e a população em geral participem da proposição de alternativas. No entanto, o relatório não aponta qual é a melhor alternativa, apenas discute os prós e contras de cada uma salientando que a melhor escolha é aquela que possa proporcionar a maior expansão 
do parque gerador hidrelétrico de modo sustentável.

$\mathrm{E}$, para isso, é preciso que se analise outras possibilidades, como a melhoria da capacidade de geração de energia nas usinas e centrais hidrelétricas, além de continuar atualizando os estudos de criação de cenários e potencial de aproveitamento de cada rio. Assim, nota-se que o desenvolvimento de alternativas da AAE do PGHMG teve cumprimento de $67 \%$ dos critérios elencados por Carvalho (2014), não estando adequado apenas aos dois últimos, sendo eles relacionados a como a alternativa preferida foi escolhida.

Tabela 5 - Avaliação da forma como as alternativas foram elaboradas nas AAE.

\begin{tabular}{l|c|c}
\hline \multicolumn{1}{c|}{ Critérios } & PRMG & PGHMG \\
\hline $\begin{array}{l}\text { Auxiliar na identificação das melhores alternativas (IAIA, 2002, segundo Carvalho, 2014) } \\
\text { existentes possíveis, mais sustentáveis e de longo prazo (Therivel, 2010, segundo } \\
\text { Carvalho, 2014) }\end{array}$ & $\begin{array}{c}\text { Não } \\
\text { cumpriu }\end{array}$ & Cumpriu \\
\hline $\begin{array}{l}\text { Oferecer oportunidades de proposição de alternativas tanto pelas autoridades ambientais } \\
\text { ou de sustentabilidade, como por outras instituições especializadas ou pelo público } \\
\text { (Therivel, 2010, segundo Carvalho, 2014) }\end{array}$ & $\begin{array}{c}\text { Não } \\
\text { cumpriu }\end{array}$ & Cumpriu \\
\hline $\begin{array}{l}\text { Descrever como as alternativas foram desenvolvidas (Therivel, 2010, segundo Carvalho, } \\
\text { 2014) }\end{array}$ & $\begin{array}{c}\text { Não } \\
\text { cumpriu }\end{array}$ & Cumpriu \\
\hline $\begin{array}{l}\text { Apresentar uma avaliação comparativa das alternativas “mais razoáveis” (Jones et al., } \\
\text { 2005, segundo Carvalho, 2014) }\end{array}$ & $\begin{array}{c}\text { Não } \\
\text { cumpriu }\end{array}$ & Cumpriu \\
\hline $\begin{array}{l}\text { Documentar como as alternativas preferidas foram escolhidas (Therivel, 2010, segundo } \\
\text { Carvalho, 2014) }\end{array}$ & $\begin{array}{c}\text { Não } \\
\text { cumpriu }\end{array}$ & $\begin{array}{c}\text { Não } \\
\text { cumpriu }\end{array}$ \\
\hline $\begin{array}{l}\text { Apresentar um resumo das razões para a seleção das alternativas consideradas, incluindo } \\
\text { qualquer dificuldade na compilação da informação requerida (EC, 2001, segundo } \\
\text { Carvalho, 2014). }\end{array}$ & $\begin{array}{c}\text { Não } \\
\text { cumpriu }\end{array}$ & $\begin{array}{c}\text { Não } \\
\text { cumpriu }\end{array}$ \\
\hline
\end{tabular}

Porém, a relevância desses dois últimos critérios pode ser discutida. Quando se fala em AAE é recorrente a sua caracterização como um instrumento que seja capaz de influenciar a tomada de decisões. Sánchez (2008) afirma que essa capacidade de influenciar seria o maior ganho da AAE. Silva (2010) ressalta que a AAE tem por objetivo identificar alternativas de maior grau de sustentabilidade. E Oliveira et al. (2009) destacam que a o papel da AAE é incluir a questão ambiental no processo decisório. No entanto, não se pode esquecer que as decisões, por serem estratégicas, serão tomadas também com base em questões da esfera política adotadas pelos governantes. Assim, o não cumprimento dos dois últimos critérios não possui uma relevância tão significativa para o desenvolvimento das alternativas nesse relatório de AAE.

No quadro geral dos resultados, segundo Pellin et al. (2011), as AAE no Brasil têm sido realizadas de maneira dissociada dos seus princípios básicos. Os relatórios analisados vêm, em grande parte, corroborar com tal informação, uma vez que os estudos foram realizados em um momento em que a maioria das decisões já havia sido tomada.

A AAE é o melhor cenário para a construção de alternativas por estar inserida em um momento em que poucas, ou nenhuma, decisão tenham sido tomadas (Sánchez, 2008). Neste ponto, a análise dos relatórios nos mostra que o estudo de alternativas foi bastante comprometido, devido ao estágio avançado de planejamento em que elas foram inseridas, principalmente nos casos das AAE do PERNMG e do PRMG.

As alternativas devem levar a escolha da opção menos nociva e capaz de atingir aos objetivos propostos (Oliveira et al.,2009), mas para que esse objetivo seja alcançado é necessário que o desenvolvimento de alternativas seja cuidadoso e abrangente. Dos relatórios avaliados, apenas o do Programa de Geração Hidrelétrica de Minas Gerais apresentou desenvolvimento de alternativas alinhado com as boas práticas e, ainda assim, não conseguiu cumprir todos os critérios apresentados por Carvalho (2014). As alternativas propostas no relatório de AAE do PGHMG conseguiram se enquadrar como estratégicas, mas no momento de sua proposição muitas decisões já haviam sido tomadas, como o setor em que haveria investimento (o de energia) e o modo pelo qual a energia seria gerada (energia hidrelétrica).

Cabe destacar que a falha no estudo de alternativas dentro das AAE não é exclusividade da prática do instrumento em Minas Gerais. Trabalhos anteriores como o de Margato \& 
Sánchez (2014) apontam que as AAE realizadas no Brasil compartilham de várias deficiências, estando entre elas a falta de análise de alternativas, bem como a participação social limitada e uma fraca influência na tomada de decisões. Ainda, o cenário mundial também remete para essa deficiência, sendo encontrada na jurisdição de Quebec no Canadá (Noble, 2004), na Austrália, Nova Zelândia, Reino Unido e Finlândia (Fischer, 2007).

\section{CONCLUSÃO}

As análises realizadas no estado de Minas Gerais levam a confirmação do relatado na literatura mundial de que a prática de $\mathrm{AAE}$ encontra-se prejudicada pelo momento em que se insere, visto que nos três relatórios boa parte das decisões já havia sido tomada quando da utilização do instrumento.

O relatório do Programa de Eletrificação Rural do Noroeste de Minas Gerais, por exemplo, foi produzido em um momento em que algumas obras do programa já estavam em andamento. Os demais programas não enfrentaram tal problema, mas foram submetidos à AAE já em estágio avançado de desenvolvimento, do ponto de vista do seu planejamento. Outro problema ainda mais frequente nos relatórios avaliados foi a falta de uma metodologia para o desenvolvimento de alternativas. No caso do Programa Rodoviário de Minas Gerais, a alternativa apresentada não passou por nenhum processo de desenvolvimento ou justificativa de proposição e escolha.

Quando a AAE realizada apresenta a proposição de alternativas, falta uma compa- ração adequada entre elas para que os tomadores de decisão possam facilmente identificar as melhores alternativas. É o caso do Programa de Geração Hidrelétrica de Minas Gerais que apresentou o desenvolvimento de alternativas estratégicas e realizou comparações entre elas, mas falhou ao não apontar claramente como chegou a melhor alternativa.

Assim, pode-se concluir que a análise de alternativas dentro do contexto de AAE ainda é deficiente em Minas Gerais por não ter um caráter estratégico e por não ser desenvolvida de acordo com critérios internacionais de boas práticas. Dessa forma, a AAE perde a sua capacidade de influenciar a tomada de decisão e passa a compor apenas um documento que acrescenta informações ao Estudo de Impacto Ambiental e ao processo de Licenciamento Ambiental, sem que tais informações sejam levadas em consideração na concessão de licenças. Esta deficiência remete a falta de regulamentação do instrumento AAE no estado e no país, aliada a uma gestão pública desinteressada e carente de comprometimento com a preservação ambiental.

\section{REFERÊNCIAS}

CARVALHO, A.F. "Timing" para uma perspectiva mais estratégica da prática de Avaliação Ambiental Estratégica. São Carlos, 2014. 320p. Tese (Doutorado em Ciências da Engenharia Ambiental). Escola de Engenharia de São Carlos Universidade de São Paulo.

EGLER, P.C.G. Perspectivas de uso no Brasil do processo de Avaliação Ambiental Estratégica. Parcerias Estratégicas, v. 6, n. 11, p. 175-190, 2011.

GANEM, R.S.; JURAS, I.A.G.M.; VIANA, M. B.; MERCADANTE, M.; ARAÚJO, S.M. V.G.; BRASILEIRO, V.M.M. Avaliação Ambiental Estratégica. Brasília: Câmara dos Deputados, 2014. Disp. em: <http://www2.camara.leg. br/documentos-epesquisa/publicacoes/estnottec/tema14/CP14 020.pdf>. Acesso em: 2 de mai de 2016.

EPA - Environmental Protection Agency. Developing and assessing alternatives in Strategic Environmental Assessment. 2015. Disp. http://www.epa.ie/pubs/advice/ea/SEA-Alternatives-157Published_web.pdf. Acesso em: 13 ago. 2016.

EC-EUROPEAN COMISSION. Directive 2001/42/EC of the European Parliament and the Council of 27 June 2001, on the assessment of the effects of certain plans and programmes on the environment. Official Journal of the European Community, 2001. In: CARVALHO, A.F.
"Timing" para uma perspectiva mais estratégica da prática de Avaliação Ambiental Estratégica. São Carlos, 2014. 320p. Tese (Doutorado em Ciências da Engenharia Ambiental). Escola de Engenharia de São Carlos - Universidade de São Paulo.

FISCHER, T.B. The theory and practice of strategic environmental assessment: towards a more systematic approach. UK/USA: Earthscan. 2007, 186p.

IAIA-International Association for Impact Assessment. Strategic Environmental Assessment: Performance Criteria. 2002. In: CARVALHO, A. F. "Timing” para uma perspectiva mais estratégica da prática de Avaliação Ambiental Estratégica. São Carlos, 2014, 320p. Tese (Doutorado em Ciências da Engenharia Ambiental). Escola de Engenharia de São Carlos, Universidade de São Paulo.

IAIA-International Association for Impact Assessment. Alternatives in Strategic Environmental Assessment of Plans and Programs: FASTIPS, 2014.

JOCE-Jornal Oficial das Comunidades Europeias (2001). Directiva 2001/42. Disp. em: https://www.apambiente.pt/_zdata/AAE/Enquadramento\%20 Legislativo/Dir2001_42.pdf. Acesso em: 13 nov. 2017.

JONES, C.; BAKER, M.; CARTER, J.; JAY, S.; SHORT, M.; WOODS, C. Strategic Environmental Assessment and land 
use planning: an international evaluation. London, UK: Earthscan, 2005. In: CARVALHO, A. F. "Timing” para uma perspectiva mais estratégica da prática de Avaliação Ambiental Estratégica. São Carlos, 2014, 320p. Tese (Doutorado em Ciências da Engenharia Ambiental). Escola de Engenharia de São Carlos, Universidade de São Paulo.

MALVESTIO, A.C. Análise da Efetividade da Avaliação Ambiental Estratégica como instrumento de política ambiental no Brasil. São Carlos, 2013. 198 p. Dissertação (Mestrado em Ciências da Engenharia Ambiental). Escola de Engenharia de São Carlos, Universidade de São Paulo.

MARGATO, V. \& SÁNCHEZ, L.E. Quality and outcomes: a critical review of Strategic Environmental Assessment in Brazil. Journal of Environmental Assessment Policy and Management, v. 16, n. 2, p. 1-32, 2014.

MINAS GERAIS. Decreto n 43.372, de 05 de junho de 2003. Cria o Núcleo de Gestão Ambiental - NGA nas Secretarias de Estado que menciona e dá outras providências. Diário Oficial do Estado de Minas Gerais, 2003.

MMA-Ministério de Meio Ambiente (Brasil). Avaliação Ambiental Estratégica. Brasília: SQA, 2002, 91p.

NOBLE, B.F. A state-of-practice survey of policy, plan, and program assessment in Canadian provinces. Environmental Impact Assessment Review, v. 24, n. 3, p. 351-361, 2004.

OLIVEIRA, I.D.; MONTAÑO, M.; SOUZA, M.P. Avaliação Ambiental Estratégica. São Carlos: Suprema, 2009, 218p.

PARTIDÁRIO, M.R. Guia de boas práticas para Avaliação Ambiental Estratégica - orientações metodológicas. Lisboa:
Agência Portuguesa do Meio Ambiente, 2007.

PELLIN, A.; LEMOS, C.C.; TACHARD, A.; OLIVEIRA, I.S.D.; SOUZA, M.P. Avaliação Ambiental Estratégica no Brasil: considerações a respeito das agências multilaterais de desenvolvimento. Engenharia Sanitária e Ambiental, v. 16, n. 1, p. 27-36, 2011.

PORTO, C. Uma introdução ao planejamento estratégico. Boletim técnico do SENAC, v. 19, n.2, p. 22-33, 1998

SÁNCHEZ, L.E. Avaliação Ambiental Estratégica e sua Aplicação no Brasil. São Paulo: Instituto de Estudos Avançados da Universidade de São Paulo, 2008.

SILVA, F.R. Avaliação Ambiental Estratégica como instrumento de promoção do desenvolvimento sustentável. Revista Direitos Fundamentais\& Democracia, v. 8, n. 8, p. 301-329, 2010.

THERIVEL, R. \& PARTIDÁRIO, M.R. The Practice of Strategic Environmental Assessment.London: Earthscan, 1996, 206p.

THERIVEL, R. Strategic Environmental in Action. Second Edi ed. London, UK: Earthscan, 2010. 366p. In: In: CARVALHO, A. F. "Timing" para uma perspectiva mais estratégica da prática de Avaliação Ambiental Estratégica. São Carlos, 2014. 320 p. Tese (Doutorado em Ciências da Engenharia Ambiental). Escola de Engenharia de São Carlos, Universidade de São Paulo.

Submetido em 7 de abril de 2017 Aceito em 5 de julho de 2018 